\title{
Molecular dynamics simulation of interface dynamics during the fcc-bcc transformation of a martensitic nature
}

\author{
C. Bos, ${ }^{*}$ J. Sietsma, and B. J. Thijsse ${ }^{\dagger}$ \\ Department of Materials Science and Engineering, Delft University of Technology, 2828 AL Delft, Netherlands
}

(Received 29 November 2005; published 30 March 2006)

\begin{abstract}
The structural and dynamic properties of the interface during the fcc-bcc transformation in pure iron have been investigated by molecular dynamics simulations. An embedded atom method potential was used for the atomic interactions. Two interfaces, close to the Bain and Kurdjumov-Sachs orientation relations, have been examined during the fcc-to-bcc transformation. In each simulation the system was left to evolve freely at the imposed temperature. In a system with fully periodic boundaries no interface motion has been observed, whereas systems with at least one free boundary do show a mobile interface. After an incubation time, there is a very fast transformation from fcc to $\mathrm{bcc}$, with interface velocities reaching values in the range of $200-700 \mathrm{~m} / \mathrm{s}$, depending on the interface orientation and on temperature. The characteristics of the transformation are of a martensitic nature, without this being imposed on the system. During the incubation time a complex interface structure is formed, which appears to be essential for the martensitic transformation. From the atomic displacements during the transformation, the occurrence of slip planes can be identified.
\end{abstract}

DOI: 10.1103/PhysRevB.73.104117

PACS number(s): $02.70 . \mathrm{Ns}, 64.70 . \mathrm{Kb}, 81.30 . \mathrm{Kf}$

\section{INTRODUCTION}

The kinetics of phase transformations in metallic alloys has been studied extensively, especially transformations that are governed by the longe-range diffusion of alloying elements. ${ }^{1}$ Also interface-controlled phase transformations ${ }^{1}$ have been the subject of numerous studies. Although a general insight into the kinetics of these diffusional phase transformations has been developed, observations on the actual atomic processes taking place at the interface during the transformation are still very scarce. ${ }^{2,3}$ The insight in the nature of martenstic transformations is even more limited than for diffusional transformations. The definition of a martensitic transformation is based on the characteristics of the atomic processes, viz. a collective motion of atoms which move over less than an interatomic distance during the process, but neither the kinetics nor the atomic processes at the interface have been investigated extensively. The reason for this lies, of course, in the experimental difficulties for such studies. No experimental technique is capable of observing the atomic motion taking place during the movement of the interface at a velocity possibly as high as the velocity of sound. ${ }^{4}$ Therefore, the scientific question on the fundamental character of the martensitic transformation remains largely unanswered, whereas this question is not only of scientific but also of great practical importance, for instance, for martensite formation in steel and in shape-memory alloys.

At present, simulation by means of molecular dynamics (MD) seems to be one of the very few methods available to acquire information about the nature of the martensitic transformation. Because the transformation is very fast, the actual transformation time can be covered in an MD-simulation. A limited number of studies applying the MD technique to martensitic transformations have been reported in the literature. Lill and Broughton ${ }^{5}$ have studied the martensitic transformation after artificially imposing the nucleation event by a particular choice of simulation conditions. The martensitic bcc-hcp transition in zirconium has been studied more extensively. ${ }^{6-8}$ Unfortunately, none of these studies are focused on the interface. Important aspects as interface structure and interface velocity are not mentioned as the focus is more on the resulting microstructure. In another study, Meyer and Entel have studied the martensite-to-austenite retransformation in iron. ${ }^{9}$ In the present study we will investigate the decomposition of austenite in iron. Austenite is the fcc phase of iron, which is stable at higher temperatures. During cooling, it is known to transform either diffusionally into ferrite or by a martensitic transformation into martensite. In this study, the simulation system consists of an fcc grain that is neighbored on two sides by a bcc grain. This system is allowed to freely evolve in time at a temperature at which the bcc phase is the stable phase. Therefore, no transformation mechanism or kinetics are imposed. The focus of this work is on the properties of the moving interface. This is also the main reason that the start configuration for the simulations already contains a stable bcc phase.

The simulations have been performed on different simulation systems, with the relative orientations of the bcc and fcc grains and the surface area as important parameters (Sec. II). By "surface" we mean the free boundaries of the system, i.e., those boundary planes that are not connected to periodic images of the system. The simulation results of the observed phase transformations (Sec. III) are discussed in terms of the interfacial structure, the free boundaries, and the influence of temperature and driving force in Sec. IV.

\section{MOLECULAR DYNAMICS}

\section{A. Johnson-Oh embedded atom method formalism}

A good description of an interface in motion requires a large three-dimensional (3D) simulation system, consisting of at least 10000-100000 atoms. Because of this requirement, the use of highly sophisticated atomic interaction 
TABLE I. Input parameters for the iron EAM potential.

\begin{tabular}{cccccc}
\hline \hline$a(\AA)$ & $E_{C}(\mathrm{eV})$ & $\Omega B(\mathrm{eV})$ & $\Omega \mu(\mathrm{eV})$ & $A$ & $E_{1 V}^{U F}(\mathrm{eV})$ \\
\hline 2.86645 & 4.29 & 12.26 & 6.53 & 2.48 & 1.75 \\
\hline \hline
\end{tabular}

schemes would lead to unfeasibly long simulation times. We have chosen a relatively simple yet sufficiently realistic interaction model, namely, the embedded atom method (EAM). ${ }^{10}$ This class of $N$-body potentials is known to function well in cases where defects are important.

The EAM, first developed by Daw and Baskes, ${ }^{11,12}$ describes the potential energy $V$ of a system as

$$
V=\frac{1}{2} \sum_{i} \sum_{j \neq i} \phi\left(r_{i j}\right)+\sum_{i} F\left(\rho_{i}\right)
$$

with

$$
\rho_{i}=\sum_{j \neq i} \rho^{a}\left(r_{i j}\right)
$$

Here $\rho_{i}$ is interpreted as the electronic charge density at the site of atom $i$, resulting from the spherically symmetric charge densities $\rho^{a}(r)$ carried by each of the neighboring atoms $j$, and $r_{i j}$ is the interatomic distance. The embedding function $F(\rho)$ describes the potential energy of an atom embedded in a given electronic charge density. The pair potential $\phi(r)$ is the two-body contribution to the potential energy. Johnson and Oh have developed an analytical EAM model for bcc metals ${ }^{10}$ in which the potential parameters are expressed as functions of seven properties of the element to be modeled. These are the cohesive energy $E_{c}$, lattice constant $a$, atomic volume $\Omega$, bulk modulus $B$, Voigt average shear modulus $\mu$, anisotropy ratio $A$, and the unrelaxed vacancy formation energy $E_{1 V}^{U F}$. The pair potential is given by

$$
\phi(r)=K_{3}\left(\frac{r}{r_{1 e}}-1\right)^{3}+K_{2}\left(\frac{r}{r_{1 e}}-1\right)^{2}+K_{1}\left(\frac{r}{r_{1 e}}-1\right)+K_{0}
$$

with $r$ the interatomic distance, $r_{1 e}$ the nearest-neighbor distance in the equilibrium bcc crystal, and $K_{0}-K_{3}$ constant parameters. The spherical charge density is expressed as

$$
\rho^{a}(r)=f_{e}\left(\frac{r_{1 e}}{r}\right)^{\beta},
$$

with $f_{e}$ a dimensionless factor that is immaterial for monoatomic potentials, and the power $\beta$ has been given the value 6 by Johnson and Oh.

The embedding function has the form

$$
F\left(\rho_{i}\right)=-\left(E_{c}-E_{1 V}^{U F}\right)\left[1-\ln \left(\frac{\rho_{i}}{\rho_{e}}\right)^{n}\right]\left(\frac{\rho_{i}}{\rho_{e}}\right)^{n},
$$

with $\rho_{e}$ the electron density at each lattice site of the equilibrium crystal, and $n$ is given by

$$
n=\sqrt{\frac{9 \Omega B-15 \Omega \mu}{\beta^{2}\left(E_{c}-E_{1 V}^{U F}\right)}} .
$$

To limit the calculation time, the potential and the spherical density function are set to zero at a cutoff distance $r_{c}$, where the value of $V$ becomes very small. This distance has been chosen as $r_{c}=r_{2 e}+\frac{1}{2}\left(r_{3 e}-r_{2 e}\right)$ with $r_{2 e}$ and $r_{3 e}$ the second and third neighbor distances. The seven input properties for iron were taken as reported by Johnson and Oh and are listed in Table I.

The key quantity for the relative stability of the phases involved in the transformation is the free-energy $G$, or more specifically, the free-energy difference between the phases. Therefore, it is important that the chosen potential describes the fcc-bcc free-energy difference well. Figure 1 shows the free-energy difference for the iron EAM potential used here, as determined with a MD adaptation of the method introduced by Miller and Reinhardt. ${ }^{13}$ It is seen that for this system the bcc phase is the stable phase. At low temperatures, the value of $\Delta G$ for the present system is similar to the experimental value for iron, as can be readily calculated from thermodynamical databases. With increasing temperature, $\Delta G$ does decrease in absolute value, as expected, although not rapidly enough to reach $\Delta G=0$ at the ferrite-austenite equilibrium temperature of $1184 \mathrm{~K}$ that is found for real iron. In fact, the bcc phase is more stable than the fcc phase over the entire temperature range considered.

A peculiar property of this EAM model of iron is the density difference between the fcc and bcc phases. In the entire temperature range considered, the equilibrium density is lower for fcc than for bec by $\sim 5 \%$. In real iron, fcc is the denser phase by a similar difference. Although we have not studied the effect of this on the transformation in detail, it is

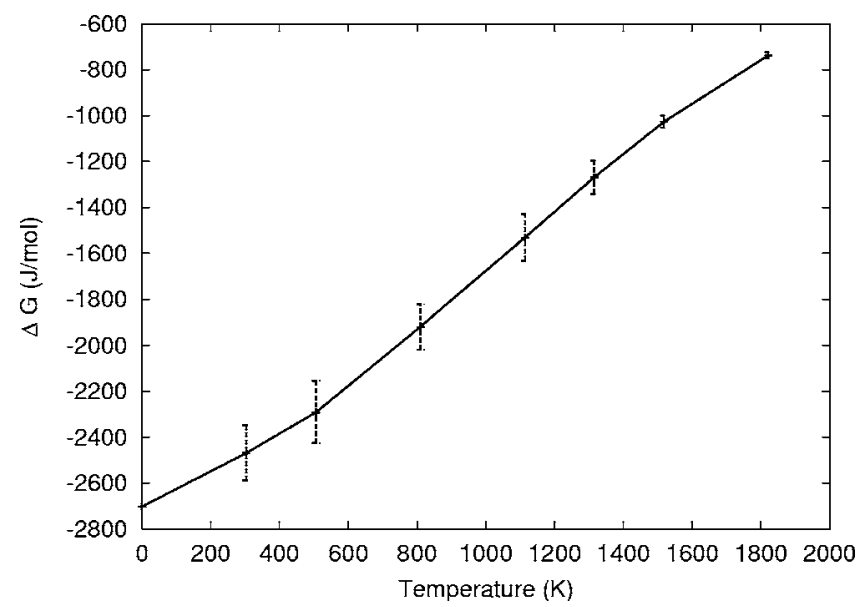

FIG. 1. The free-energy difference between fcc and bcc for the Johnson-Oh ${ }^{10}$ iron potential at zero pressure. 


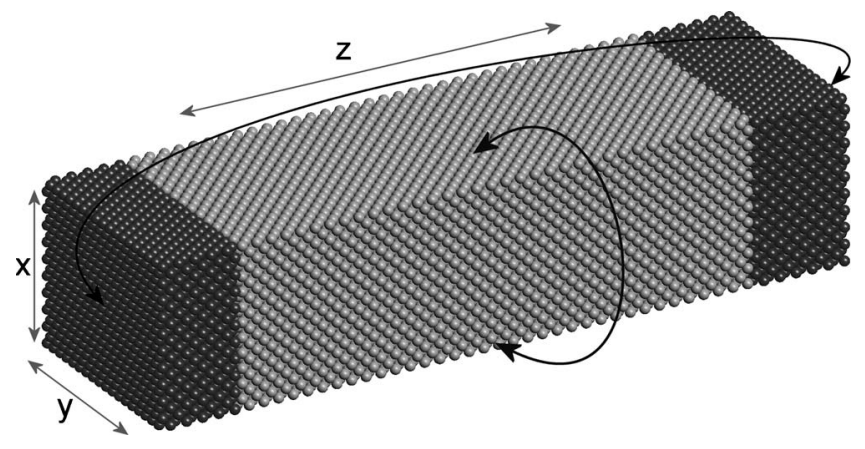

FIG. 2. A typical starting configuration for interface type $\mathrm{C}$, a system with only one nonperiodic direction (the $y$ direction). Bcc atoms are dark; fcc atoms are light. The $x$ direction in the figure coincides with the fcc [100] direction, $y$ with fcc [010], and $z$ with fcc $[001]$.

felt that the magnitude of the density difference is much more important than its sign, since with either sign local strains will develop during the transformation.

\section{B. Simulation conditions}

All simulations have been performed at zero pressure and at constant temperature, using a barostat and a thermostat of the Berendsen type. ${ }^{14}$ The MD time step was not fixed but was determined by a maximum displacement criteron of $0.02 \AA$ per time step.

The simulations of the fcc-to-bcc transformation have been performed with systems of different sizes, and with periodic boundaries in either one, two, or three directions. The simulation box (Fig. 2) was rectangular, and the system always contained two bcc/fcc interfaces perpendicular to the $z$ direction, in which periodic boundary conditions were applied in all cases. Table II gives an overview of all the interface variations that have been examined.

Interface types $\mathrm{A}, \mathrm{B}$, and $\mathrm{C}$ have an fcc $\{100\} \|$ bcc $\{110\}$, fcc $\langle 100\rangle \|$ bcc $\langle 011\rangle$ Bain orientation relation, and interface type D has an fcc $\{111\} \| \operatorname{bcc}\{110\}$, fcc $\langle 112\rangle \| \operatorname{bcc}\langle 011\rangle$ orien-

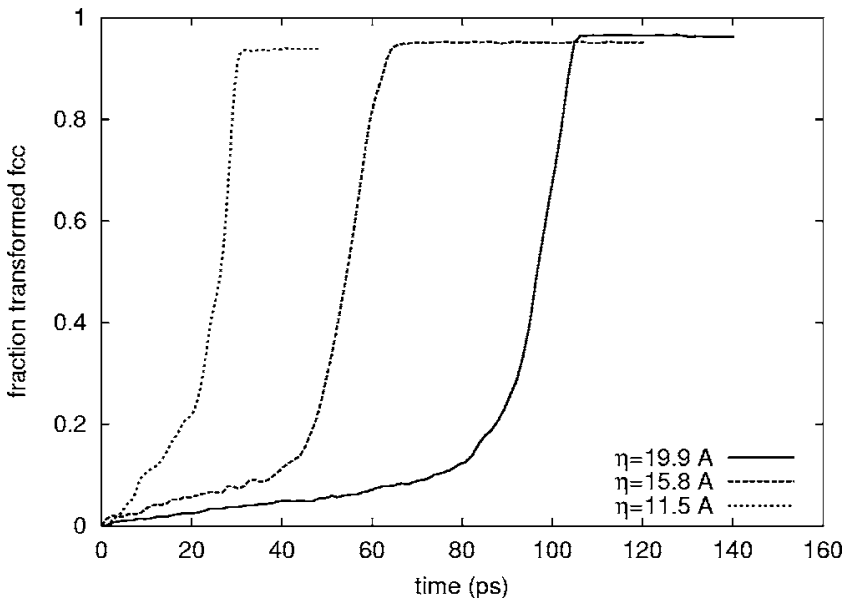

FIG. 3. Typical transformation curves for interface type B at $T$ $=810 \mathrm{~K}$, for different values of $\eta$.

tation relation, which is close to the Kurdjumov-Sachs orientation relation. The difference between $\mathrm{A}, \mathrm{B}$, and $\mathrm{C}$ is the number of periodic directions. An important characteristic of the systems, also reported in Table II, is the volume to surface area ratio $\eta$. Figure 2 shows a typical starting configuration for interface type $\mathrm{C}$.

The starting configurations were constructed by generating fcc and bcc crystals at their own equilibrium densities and bringing them together at a distance equal to the interplanar spacing. The lattice parameter of the fcc and bcc parts had to be slightly adapted $(\approx 0.2 \%$, with opposite signs for the two phases) to create a fit within the common periodic boundaries. Simulations with an explicit relaxation time period (realized by a very slow warm-up to the required temperature) showed no difference in behavior in comparison to simulations without this relaxation period. The thermostat quickly removes the excess energy of the atoms that have an unphysically strong interaction in the initial unrelaxed structure.

At any moment during the simulations each atom is determined to be in an fcc or a bcc configuration by consider-

TABLE II. Overview of all interfaces types, temperatures, and system sizes that have been simulated, as well as the volume to surface area ratio $\eta$ for each system.

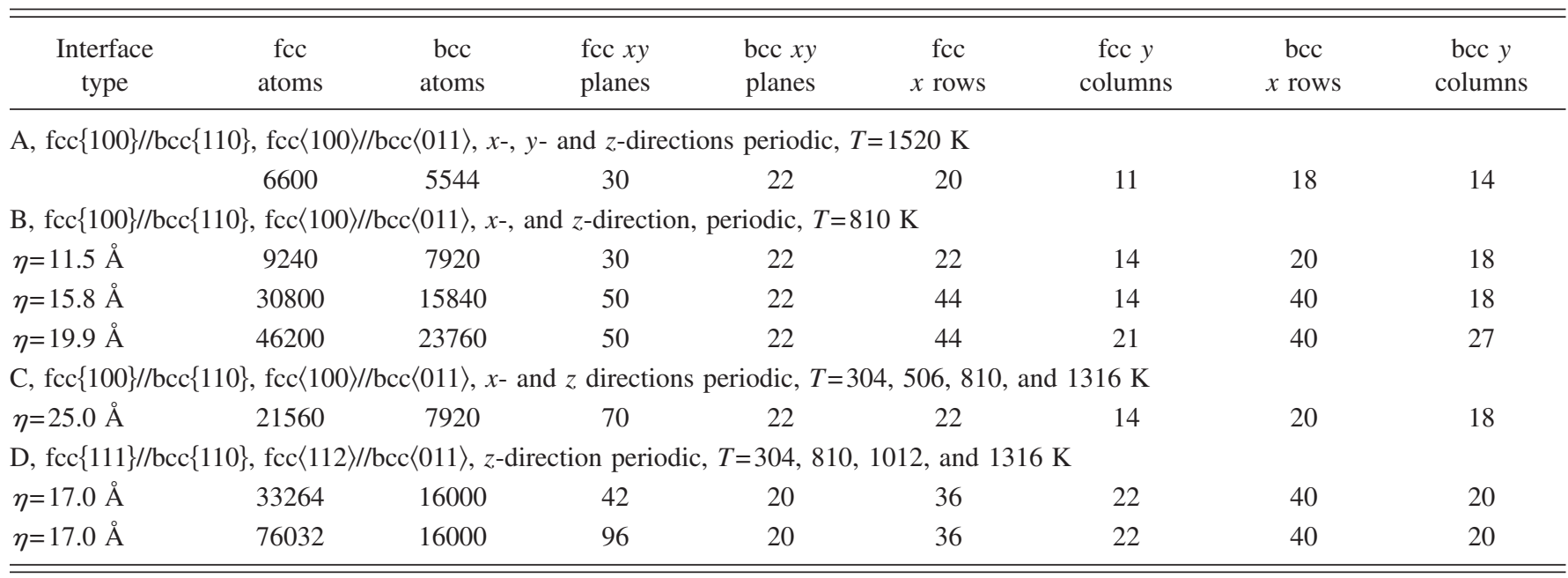




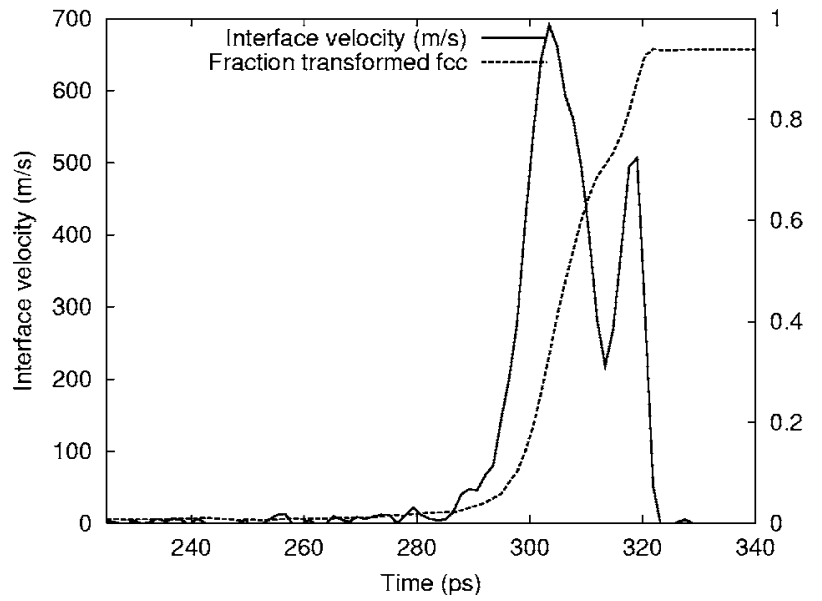

FIG. 4. Typical transformation curve for interface type $\mathrm{C}$ at $T$ $=304 \mathrm{~K}$.

ing the locations of its nearest neighbors averaged over $1.4 \mathrm{ps}$, described in terms of angles of atom triplets. This procedure is based on rotationally invariant spherical harmonics as proposed in Ref. 15.

\section{RESULTS}

\section{A. Simulation system A}

After a simulation time of $8.6 \mathrm{~ns}$ at $T=1520 \mathrm{~K}$ in system $\mathrm{A}$, about 0.15 monolayers of the initially fcc-configured atoms have transformed to bcc, but after that the bcc phase does not grow. Even after 48 ns there is no significant increase in the fraction of bcc atoms. Simulations at different temperatures show the same behavior: a small increase in the number of bcc-configured atoms in the initial stage and no subsequent phase transformation.

Close examination of the atomic configurations at the interface shows that the structure of the fcc and bcc planes that make up the interface changes in a very brief time span, much shorter than the $8.6 \mathrm{~ns}$ mentioned before. A perfect bcc $\{110\}$ plane in a system of these dimensions contains 252

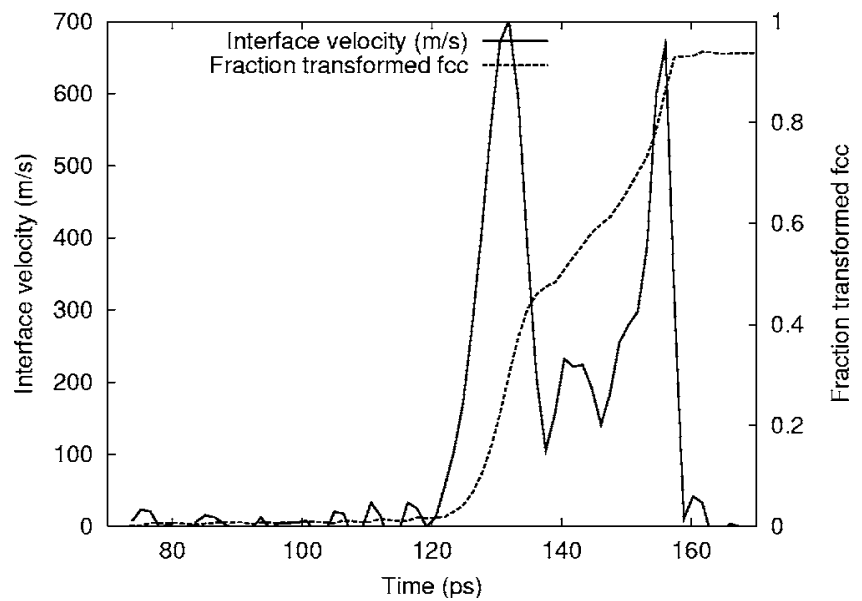

FIG. 5. Typical transformation curve for interface type $\mathrm{C}$ at $T$ $=506 \mathrm{~K}$.

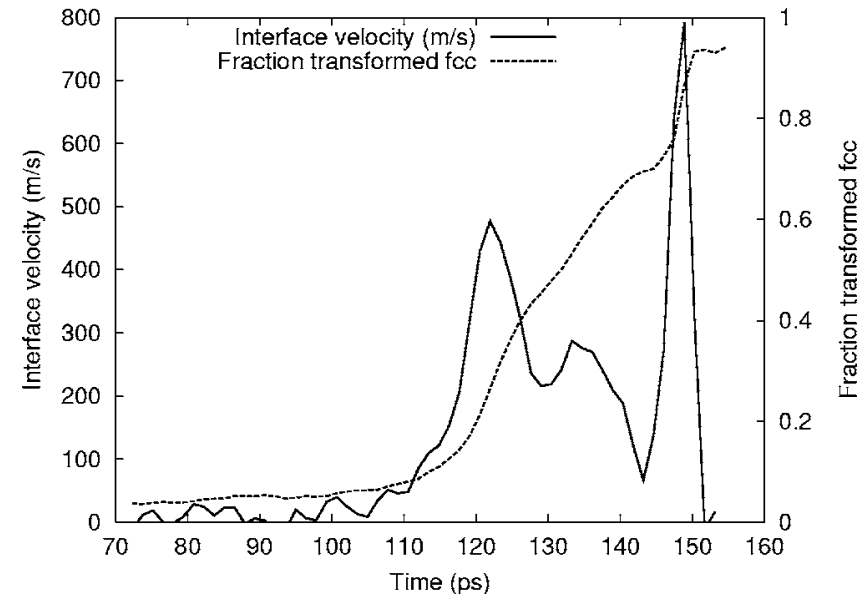

FIG. 6. Typical transformation curve for interface type $\mathrm{C}$ at $T$ $=810 \mathrm{~K}$.

atoms and a perfect fcc $\{100\}$ plane contains 220 atoms. After $68 \mathrm{ps}$ at $1520 \mathrm{~K}$, the fcc plane has acquired seven atoms from the adjacent bcc plane. Consequently, both planes contain a relatively large amount of free space in comparison to a perfect bcc $\{110\}$ plane. The free space in these interfacial planes is not present in the form of vacancies or dislocations but rather in the form of density inhomogeneities within the plane. The atomic structure in the two interfacial planes is partially disordered. Occasionally, vacancies do form and diffuse into the bec crystal.

\section{B. Simulation systems B, C, and D}

Figures 3-8 show that for systems with at least one free boundary, the fcc phase transforms into bcc on a time scale of tens of picoseconds for the present system dimensions. After a certain incubation time, in which the transformation proceeds relatively slowly over a few monolayers at each interface, one or both of the interfaces start to move very rapidly up to complete transformation. Although the presence of a free boundary turns out to be essential for the transformation to take place, primarily because of the density

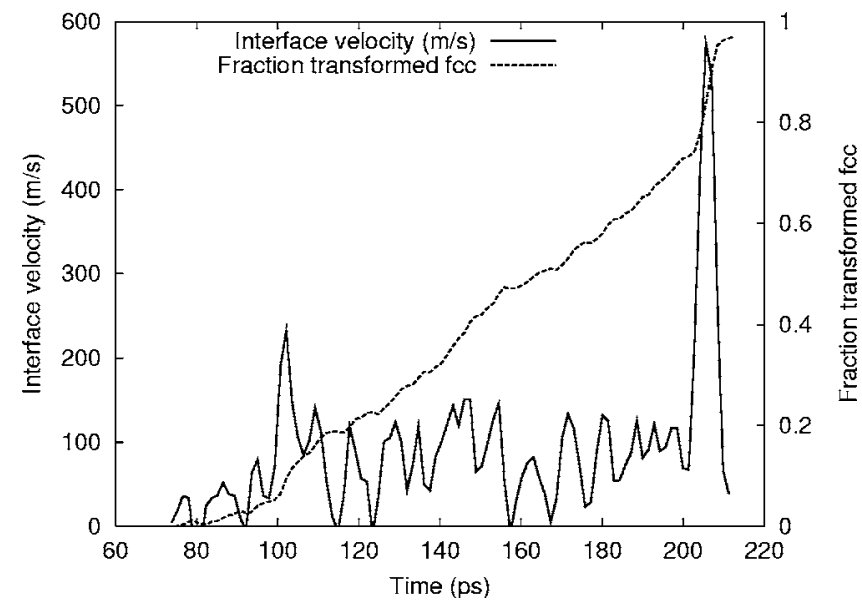

FIG. 7. Typical transformation curve for interface type $\mathrm{C}$ at $T$ $=1316 \mathrm{~K}$. 


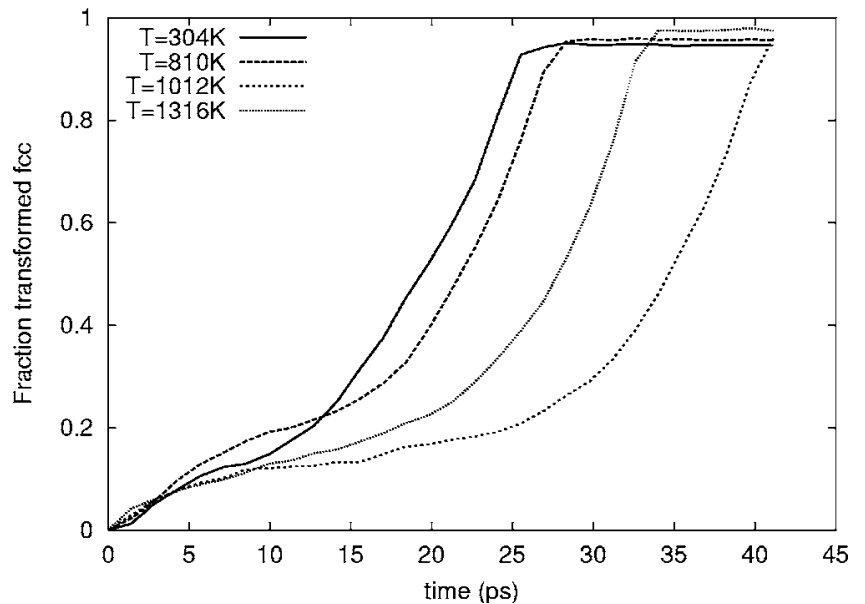

FIG. 8. Transformation curves at four temperatures. Interface type D.

difference of the phases, the transformation kinetics also depend on the type of interface, the ratio $\eta$, and the temperature.

Figure 3 shows that the incubation time is longer for a larger $\eta$. This can be explained by the larger absolute misfit that has to accommodated at the free surface. The relative strain $(0.2 \%)$ has no influence, since it is independent of $\eta$.

All systems require a certain incubation time before the transformation starts. During this period the atomic structure at the interface changes, and when the interface motion starts, all interface types show a structure that appears to be of a universal character. Figure 9 shows an example. To enhance the level of detail, the scale in the $y$ direction has been elongated by a factor of 7 . The results for the different interfaces indicate that the interface type plays an important role

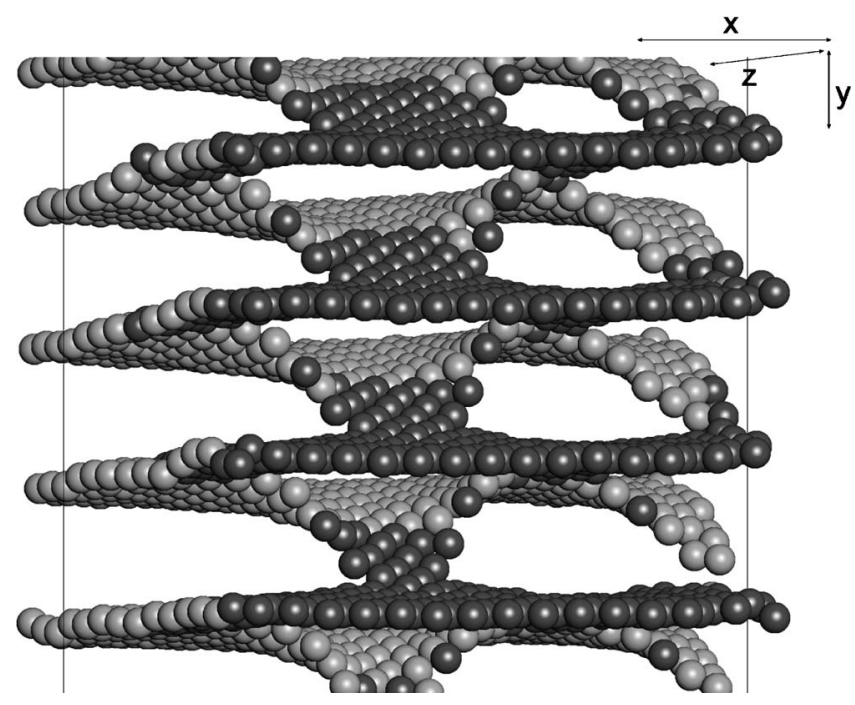

FIG. 9. Close up of the structure at the interface, just before the interface motion starts, for interface type $\mathrm{C}$ at $T=810 \mathrm{~K}$. The interface clearly shows a close resemblance to a network of screw dislocations. Dark atoms are bcc; lighter atoms are fcc. The $y$ coordinates have been multiplied with a factor 7. The $x$ direction in the figure coincides with the fcc [100] direction, $y$ with fcc [010], and $z$ with fcc [001]. in the temporal development of the interface structure. This type of interface structure, which is formed by gradual motion of the atoms during the incubation time, must necessarily be formed across the entire interface before the interface can start to move. The interface clearly shows a close resemblance to a network of screw dislocations. This is corroborated by the slip mechanism that is active during the transformation (see Fig. 10). Figures 4-7 suggest that the formation of the interface structure required for transformation is related to the driving force: the incubation time is found to be inversely proportional to $\Delta G$, and the temperature dependence of the proportionality constant is given by an effective activation energy of $0.06 \mathrm{eV}$.

The deformation index ${ }^{16} u_{i}$ for atom $i$ is defined as

$$
u_{i}=\max \left(\left|\mathbf{r}_{i j}-\mathbf{r}_{i j}^{0}\right|\right) \text {, }
$$

where $j$ represents all nearest neighbors of atom $i$ in the initial atomic configuration, $\mathbf{r}_{i j}$ is the vector between atoms $i$ and $j$ in the final configuration, and $\mathbf{r}_{i j}^{0}$ is the same vector in the initial configuration. The deformation index is therefore the maximum relative displacement of an atom with respect to its nearest neighbors. Most atoms in the system have a deformation index that is distinctly smaller than the interatomic distance; for these atoms the transformation takes place by means of small atomic displacements, a picture that is usually connected to a martensitic transformation. These displacements have been observed in the present study to be highly coordinated. In Fig. 10, the atoms are shown that have a larger deformation index, viz. between 2.4 and $2.5 \AA$, a distance close to the interatomic distance. From this figure it can be concluded that in addition to the small displacements for most atoms, atomic displacements on the order of an interatomic distance occur along certain planes. These planes can be recognized as $\{111\}$ planes in the fcc structure and $\{110\}$ planes in the bcc structure. Note that the same atoms are depicted in both frames of Fig. 10. The figure therefore shows that the transformation is accompanied by dislocation glide, since each set of two parallel planes can be understood to consist of the atomic planes on either side of a slip plane. The principal reason for the dislocation glide is in the stresses caused by the phase transformation in combination with the constraints on the system because of the periodic boundary conditions in the $z$ direction. Figure 10 also shows that an orientation relation exists between the parent phase and the newly formed phase, according to which the closest packed planes in both structures are parallel (consistent with the Kurdjumov-Sachs and Nishiyama-Wasserman orientation relations, which were not imposed on the system by the initial configuration).

Figure 3 shows that the $\eta$ ratio only has an influence on the incubation time, but not on the transformation rate. On the other hand, the temperature does influence the maximum interface velocity, as shown in Fig. 11 for system C.

The linear decrease of the interface velocity with increasing temperature indicates that the velocity is not determined by a thermally activated process, but rather by the freeenergy difference, which linearly decreases with increasing temperature (Fig. 1), acting as a driving force for the transformation. 

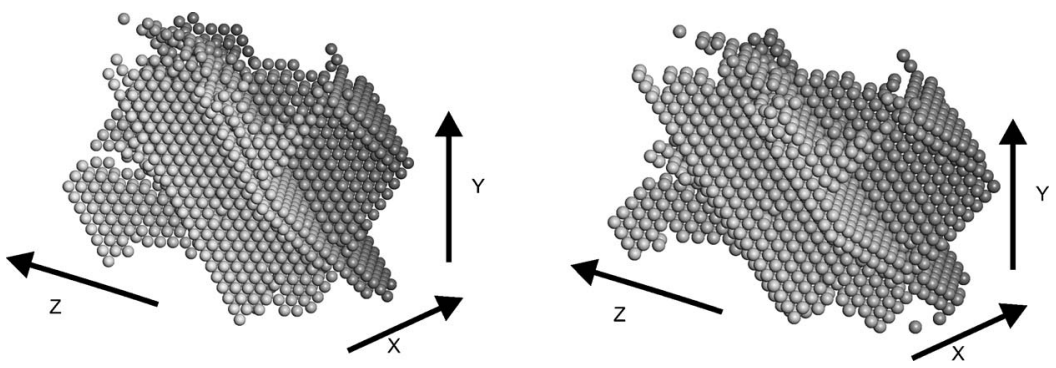

FIG. 10. The slip planes of the dislocations shown in the initial fcc structure (left) and in the final structure (right). Only the atoms with a deformation index between 2.4 and $2.5 \AA$ are shown.

\section{DISCUSSION}

The fcc-bcc interfaces in simulation system A are not mobile. As mentioned in Sec. III A, in simulation system A an fcc $\{100\}$ plane contains 220 atoms. If the fcc phase would transform into the bcc phase by, for example, a Bain distortion of the lattice, the resulting bcc plane would again contain 220 atoms. The ratio of the width and height of the plane changes as this transformation takes place. However, if periodic boundaries are used, this change is not possible and any new bcc plane must take the shape and size (and therefore also the same number of atoms) of the bcc planes already present. Even when the more "flexible" Parrinello-Rahman ${ }^{17}$ periodic boundary conditions are used, the already present bcc phase will prohibit the required shape change of the simulation volume. Because the periodic boundary conditions prohibit a transformation by a single collective motion of the atoms, the only alternative mechanism left is a diffusional transformation. That such a transformation does not take place must be ascribed to the density difference between the two phases. Each new bcc place resulting from a diffusional transformation must contain 252 atoms. The extra 32 atoms can only be acquired by the formation of vacancies in other parts of the system. The formation of such an extraordinary large concentration of vacancies during the present simulation times is extremely unlikely.

With the introduction of a free surface as in simulation systems B, C, and D, both (diffusional and martensitic) transformation mechanisms can be more easily established. The surface can readily accommodate the density difference and will also allow a shape change of the crystal volume.

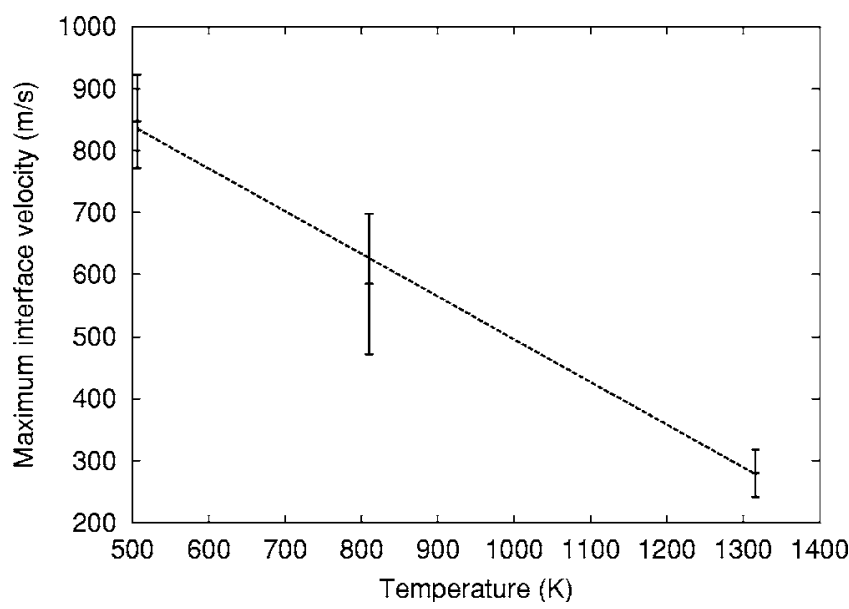

FIG. 11. Maximum interface velocity as a function of temperature, for interface type C.
Although the details of the transformation mechanism for the three systems with their different types of interface are different, they share many characteristics. Two of those characteristics also belong to typical martensitic transformations. The first is the very high interface velocity; the second is the coordinated, but very small, movement of the majority of atoms during the transformation. Besides these small atomic displacements, a slip mechanism occurs along closely packed planes in both crystalline structures (see Fig. 10).

The question whether or not a transformation will take place in a more realistic system, in which many 3D grains are present and open grain boundaries may act as a source for absorbing density differences, remains unanswered. Future work on this subject is planned.

The temperature and the volume-to-surface area ratio have a large influence on the formation of the specific interface structure during the incubation time (Fig. 9). The scatter in the incubation times of replica runs makes it very difficult to find an accurate relationship between these two parameters and the formation of the interface structure. Nevertheless, the present simulations indicate that the temperature dependence of this process is primarily determined by the thermodynamic driving force $\Delta G$.

Temperature does not only influence the incubation time, but also the maximum interface velocity. With increasing temperature the maximum interface velocity decreases considerably, although the transformation mechanism does not seem to change. The underlying reason for this behavior is again the free-energy difference $\Delta G$, which decreases linearly with temperature (Fig. 1). It therefore appears that for this martensitic transformation, similar to the role of the driving force during interface-controlled diffusional transformations, ${ }^{1,18}$ the interface velocity is also proportional to the driving force. The proportionality constant, the interfacial mobility, assumes a very high value, i.e., approximately $0.3 \mathrm{molm} / \mathrm{Js}$, as compared to mobilities on the order of $10^{-7} \mathrm{molm} / \mathrm{Js}$ found for diffusional austenite to ferrite transformations. ${ }^{19}$

\section{CONCLUSION}

The fcc-bcc interface is immobile in a system with full periodic boundary conditions, but moves very rapidly in systems with at least one free boundary. For both the fcc $\{100\} \|$ bcc $\{110\}$ and the fcc $\{111\} \|$ bcc $\{110\}$ interface orientations, the same kind of transformation mechanism has been found. The following picture is obtained: during an incubation time, the duration of which depends on the 
temperature, a specific interface structure is formed. Once the required interface structure has been formed, the transformation proceeds with martensiticlike characteristics. The movement of the atoms during the transformation is highly coordinated, over a small distance. In addition, slip occurs along closely packed crystallographic planes.

The temperature dependence of the maximum interface velocity is related to the temperature dependence of the freeenergy difference, which acts as the driving force for the transformation. The approximate proportionality between interface velocity and driving force indicates an interface mobility of $\sim 0.3 \mathrm{molm} / \mathrm{Js}$, which is several orders of magnitude larger than experimentally found for the diffusional austenite to ferrite transformation.
* Present address: Max Planck Institute for Metals Research, Heisenbergstrasse 3, D-70569, Stuttgart, Germany.

${ }^{\dagger}$ E-mail address: b.j.thijsse@tnw.tudelft.nl

${ }^{1} \mathrm{~J}$. W. Christian, The Theory of Transformations in Metals and Alloys (Pergamon Press, London, 2002).

${ }^{2}$ J. M. Howe, W. T. R. Reynolds, Jr., and V. K. Vasudevan, Metall. Mater. Trans. A 33A, 2391 (2002).

${ }^{3}$ T. Furuhara, K. Wada, and T. Maki, Metall. Mater. Trans. A 26A, 1971 (1995).

${ }^{4}$ R. F. Bunshah and R. F. Mehl, Trans. AIME 197, 1251 (1953).

${ }^{5}$ J. V. Lill and J. Q. Broughton, Phys. Rev. B 63, 144102 (2001).

${ }^{6}$ U. Pinsook and G. J. Ackland, Phys. Rev. B 58, 11252 (1998).

${ }^{7}$ U. Pinsook and G. J. Ackland, Phys. Rev. B 62, 5427 (2000).

${ }^{8}$ J. R. Morris and K. M. Ho, Phys. Rev. B 63, 224116 (2001).

${ }^{9}$ R. Meyer and P. Entel, Phys. Rev. B 57, 5140 (1998).

${ }^{10}$ R. A. Johnson and D. J. Oh, J. Mater. Res. 4, 1195 (1989).
${ }^{11}$ M. S. Daw and M. I. Baskes, Phys. Rev. Lett. 50, 1285 (1983).

${ }^{12}$ M. S. Daw and M. I. Baskes, Phys. Rev. B 29, 6443 (1984).

${ }^{13}$ M. A. Miller and W. P. Reinhardt, J. Chem. Phys. 113, 7035 (2000).

${ }^{14}$ H. J. C. Berendsen, J. P. M. Postma, W. F. van Gunsteren, A. DiNola, and J. R. Haak, J. Chem. Phys. 81, 3684 (1984).

${ }^{15}$ P. J. Steinhardt, D. R. Nelson, and M. Ronchetti, Phys. Rev. B 28 , 784 (1983).

${ }^{16}$ M. Wen, S. Fukuyama, and K. Yokogawa, Comput. Mater. Sci. 30, 217 (2004).

${ }^{17}$ M. Parrinello and A. Rahman, J. Appl. Phys. 52, 7182 (1981).

${ }^{18}$ Y. van Leeuwen, T. A. Kop, J. Sietsma, and S. van der Zwaag, J. Phys. IV 9, 401 (1999).

${ }^{19}$ J. J. Wits, T. A. Kop, Y. van Leeuwen, J. Sietsma, and S. van der Zwaag, Mater. Sci. Eng., A 283, 234 (2000). 